\title{
CURRENT EPIDEMIOLOGICAL KNOWLEDGE ABOUT THE ROLE OF FLAVONOIDS IN PROSTATE CARCINOGENESIS
}

\author{
K. Sak* \\ NGO Praeventio, Näituse 22-3, Tartu 50407, Estonia
}

\begin{abstract}
Numerous experimental studies have demonstrated anticancer action of polyphenolic plant metabolites. However, data about associations between dietary intake of plant-derived flavonoids and prostate cancer risk are still sparse and inconsistent. This minireview compiles the epidemiological findings published to date on the role of flavonoids in prostate tumorigenesis, discusses the reasons of inconsistencies and elicits the promising results for chemoprevention of this malignancy. Long-term consumption of high doses of soy isoflavones can be the reason of markedly lower clinically detectable prostate cancer incidence among Asian men compared to their counterparts in the Western world. The ability to metabolize daidzein to equol, the most biologically active isoflavone, by the certain intestinal bacteria also seems to contribute to this important health benefit. The increasing incidence rate of prostate cancer related to adoption of westernized lifestyle and dietary habits makes the issue of chemoprevention ever more important and directs the eyes to specific food components in the Eastern diet. If further large-scale epidemiological studies will confirm the protective effects of isoflavones against prostate cancer, this could provide an important way for prostate cancer prevention, as diet is a potentially modifiable factor in our behavioral pattern.
\end{abstract}

Key Words: chemoprevention, epidemiological findings, flavonoids, plant metabolites, prostate cancer.

The incidence rate of clinically detectable prostate cancer is historically markedly lower in Asian countries, such as Japan, China, India, Singapore or Korea, compared to the Western world, including men living in American, European countries or Canada [1-9]. This age-adjusted difference between Asian and Western populations can reach even 10- to 90-fold [10-12]. However, in parallel to adoption of westernized habits among Japanese men, the incidence rate of prostate tumors is gradually rising $[1,6,7,9]$. Also, Japanese migrants to the United States as well as foreign-born Asian Americans reveal an increased incidence of prostate neoplasms [3, 6, 10, 11, 13-16]. On the contrary, the incidence of clinically undetectable or latent prostate cancers in autopsy studies is rather similar between men from Asian and American countries, suggesting that some components in the Asian diet can block progression of latent cancers to the more advanced form [3, 6, $8,14]$. Therefore, the etiology of prostate tumor might involve environmental factors, such as dietary habits, which can play a major role in the pathogenesis of this malignancy [1, 3-6, 11-20]. A traditional Asian diet is rich in soy-derived phytoestrogens or isoflavones that have been related to the lower incidence of prostate cancer in this region [3, 7-9, 14, 21].

Plant-based food items contain various phytochemicals, including flavonoids, with anticarcinogenic properties [18]. These polyphenolic compounds may influence prostate tumorigenesis through multiple different mechanisms; flavonoids have been shown to exhibit antioxidant, antiinflammatory, antiproliferative, proapoptotic, antiangiogenic and antimetastatic activities [13, 18, 22]. Although numerous experimental cell culture and animal studies have demonstrated different effects of these plant metabolites against prostate cancer, findings from epidemiological studies

Submitted: March 13, 2017.

*Correspondence: E-mail: katrin.sak.001@mail.ee are still limited and far from definite $[6,12,16,19,21$, 23-26]. To comprehend the current state of epidemiological evidence on the role of various flavonoids on prostate tumorigenesis, the findings published to date about associations between dietary intake of flavonoids and the risk of prostate cancer were compiled and are analyzed in this minireview. In addition, the blood and urinary biomarker data are included in the survey. These data are presented in Tables 1-3. Also, the potential factors for inconsistencies are put forward and discussed.

\section{EPIDEMIOLOGICAL DATA ABOUT THE ROLE OF FLAVONOIDS ON PROSTATE CANCER RISK}

\section{Case-control studies}

No statistically significant associations with the risk of prostate cancer were found for total flavonoids or flavonoid subclasses, including flavones, flavonols, flavanones, flavanols, isoflavones and anthocyanidins in Italian population, showing no protective effects of flavonoids against prostate tumorigenesis [21]. These results were in general agreement with two other casecontrol studies, revealing no significant relationships between intake of flavones (apigenin, luteolin) and flavonols (kaempferol, myricetin) and prostate cancer incidence among American men [18, 27]. However, a suggestive inverse association was still reported for flavonol quercetin with a $36 \%$ decrease in prostate cancer risk for men in the highest quartile of quercetin consumption compared to those in the lowest quartile of intake [18].

Similarly to the work with Italian men, no overall relationships between consumption of total or individual isoflavones (genistein, daidzein) and prostate cancer risk were observed also in Swedish [17, 28], Scottish [5], or English population [12, 29]. However, these results are different from the findings of case-control studies conducted with Japanese [1] and Chinese men [15], which observed a protective effect of intake 
Table 1. Epidemiological case-control studies on dietary intake of flavonoids and prostate cancer risk

\begin{tabular}{|c|c|c|c|c|c|c|c|c|c|c|}
\hline $\begin{array}{l}\text { Flavonoid } \\
\text { subclass }\end{array}$ & $\begin{array}{c}\text { Certain } \\
\text { compound }\end{array}$ & Study ${ }^{\mathrm{a}}$ & $\begin{array}{l}\text { Popula- } \\
\text { tion }\end{array}$ & $\begin{array}{l}\text { Con- } \\
\text { trols }^{\text {b }}\end{array}$ & Cases/controls & $\begin{array}{c}\text { Intake comparison } \\
(\text { low vs high, } \mathrm{mg} / \text { day })^{\mathrm{c}}\end{array}$ & $\begin{array}{c}\text { Multivariate- } \\
\text { adjusted OR/RR }\end{array}$ & $\begin{array}{c}p \text { for } \\
\text { trend }\end{array}$ & $\begin{array}{l}\text { Age, } \\
\text { years }\end{array}$ & $\begin{array}{l}\text { Refe- } \\
\text { rence }\end{array}$ \\
\hline Flavonoids & & & Italian & $\mathrm{HB}$ & $1294 / 1451$ & (Q5) & $1.20(0.92-1.58)$ & 0.44 & $46-74$ & [21] \\
\hline Flavones & & & Italian & HB & $1294 / 1451$ & (Q5) & $1.09(0.85-1.40)$ & 0.88 & $46-74$ & [21] \\
\hline Flavones & Apigenin & & American & HB & $83 / 107$ & & $0.83(0.45-1.51)$ & 0.54 & & [27] \\
\hline Flavones & Luteolin & & American & HB & $83 / 107$ & & $0.83(0.45-1.51)$ & 0.54 & & [27] \\
\hline Flavonols & & & Italian & $\mathrm{HB}$ & $1294 / 1451$ & (Q5) & $1.23(0.95-1.61)$ & 0.26 & $46-74$ & [21] \\
\hline Flavonols & Kaempferol & WNYDS & American & PB & $433 / 538$ & $<1.4475$ vs $>6.0568$ (Q4) & $0.83(0.58-1.18)$ & 0.80 & & [18] \\
\hline Flavonols & Kaempferol & & American & HB & $83 / 107$ & & $0.77(0.42-1.43)$ & 0.41 & & [27] \\
\hline Flavonols & Myricetin & & American & $H B$ & $83 / 107$ & & $1.12(0.61-2.06)$ & 0.70 & & [27] \\
\hline Flavonols & Quercetin & WNYDS & American & $\mathrm{PB}$ & $433 / 538$ & $<10.5663$ vs $>25.5626$ (Q4) & $0.64(0.44-0.92)$ & 0.15 & & [18] \\
\hline Flavonols & Quercetin & & American & $\mathrm{HB}$ & $83 / 107$ & & $0.96(0.53-1.76)$ & 0.90 & & [27] \\
\hline Flavanones & & & Italian & HB & $1294 / 1451$ & (Q5) & $0.96(0.75-1.23)$ & 0.56 & $46-74$ & [21] \\
\hline Flavanols & & & Italian & HB & $1294 / 1451$ & (Q5) & $1.30(1.01-1.69)$ & 0.48 & $46-74$ & [21] \\
\hline Isoflavones & & EPIC-Norfolk & English & PB & $203 / 800$ & & $0.89(0.72-1.11)$ & 0.29 & $40-79$ & [29] \\
\hline Isoflavones & & & Italian & $H B$ & $1294 / 1451$ & (Q5) & $0.98(0.76-1.26)$ & 0.34 & $46-74$ & [21] \\
\hline Isoflavones & & PCANDIET & Scottish & PB & $433 / 483$ & $<0.5811$ vs $>1.9828$ (Q4) & $1.18(0.79-1.75)$ & 0.87 & $50-74$ & [5] \\
\hline Isoflavones & & CAPS & Swedish & $\mathrm{PB}$ & $1499 / 1130$ & 0.0008 vs 0.113 (Q4) & $0.99(0.77-1.28)$ & 0.68 & $36-79$ & [17] \\
\hline Isoflavones & & CAPS & Swedish & PB & $1431 / 1081$ & 0.0008 vs 0.113 (Q4) & $0.99(0.77-1.28)$ & 0.95 & $35-79$ & [28] \\
\hline Isoflavones & & & Japanese & $\mathrm{HB}$ & $200 / 200$ & $<30.5$ vs $\geqslant 89.9(\mathrm{Q} 4)$ & $0.42(0.24-0.72)$ & $<0.01^{*}$ & $59-73$ & [1] \\
\hline Isoflavones & Genistein & & American & $\mathrm{HB}$ & $83 / 107$ & & $0.71(0.39-1.30)$ & 0.26 & & [27] \\
\hline Isoflavones & Genistein & EPIC-Norfolk & English & PB & $203 / 800$ & & $0.90(0.74-1.10)$ & 0.31 & $40-79$ & [29] \\
\hline Isoflavones & Genistein & EPIC-Norfolk & English & PB & $89 / 178$ & & $1.07(0.84-1.37)$ & 0.575 & $45-75$ & [12] \\
\hline Isoflavones & Genistein & CAPS & Swedish & PB & $1499 / 1130$ & 0.00019 vs 0.0694 (Q4) & $0.97(0.75-1.26)$ & 0.09 & $36-79$ & [17] \\
\hline Isoflavones & Genistein & & Chinese & $\mathrm{PB}$ & $133 / 265$ & $<17.9$ vs $>62.0(\mathrm{Q} 4)$ & $0.53(0.29-0.97)$ & 0.058 & $50-89$ & [15] \\
\hline Isoflavones & Genistein & & Japanese & HB & $200 / 200$ & $<1.1 v s \geqslant 2.5(\mathrm{Q} 4)$ & $0.58(0.34-0.97)$ & $0.04^{\star}$ & $59-73$ & [1] \\
\hline Isoflavones & Daidzein & & American & HB & $83 / 107$ & & $0.57(0.31-1.05)$ & 0.07 & & [27] \\
\hline Isoflavones & Daidzein & EPIC-Norfolk & English & $\mathrm{PB}$ & $203 / 800$ & & $0.92(0.69-1.22)$ & 0.56 & $40-79$ & [29] \\
\hline Isoflavones & Daidzein & EPIC-Norfolk & English & PB & & & $1.06(0.84-1.64)$ & 0.630 & $45-75$ & [12] \\
\hline Isoflavones & Daidzein & CAPS & Swedish & PB & $1499 / 1130$ & 0.00037 vs 0.0431 (Q4) & $1.22(0.92-1.62)$ & 0.70 & $36-79$ & [17] \\
\hline Isoflavones & Daidzein & & Chinese & $\mathrm{PB}$ & $133 / 265$ & $<10.0$ vs $>36.3(Q 4)$ & $0.56(0.31-1.04)$ & 0.116 & $50-89$ & {$[15]$} \\
\hline Isoflavones & Daidzein & & Japanese & HB & $200 / 200$ & $<0.8 v s \geqslant 1.9(\mathrm{Q} 4)$ & $0.55(0.32-0.93)$ & $0.02^{*}$ & $59-73$ & [1] \\
\hline Isoflavones & Biochanin A & & American & $\mathrm{HB}$ & $83 / 107$ & & $0.92(0.50-1.70)$ & 0.79 & & [27] \\
\hline Isoflavones & Biochanin A & EPIC-Norfolk & English & PB & $203 / 800$ & & $0.89(0.69-1.14)$ & 0.34 & $40-79$ & [29] \\
\hline Isoflavones & Formononetin & & American & $H B$ & $83 / 107$ & & $0.99(0.54-1.81)$ & 0.98 & & [27] \\
\hline Isoflavones & Formononetin & EPIC-Norfolk & English & $\mathrm{PB}$ & $203 / 800$ & & $0.97(0.83-1.13)$ & 0.70 & $40-79$ & [29] \\
\hline Isoflavones & Glycitein & EPIC-Norfolk & English & PB & $203 / 800$ & & $0.88(0.67-1.15)$ & 0.34 & $40-79$ & [29] \\
\hline Isoflavones & Equol & EPIC-Norfolk & English & $\mathrm{PB}$ & $203 / 800$ & & $1.31(1.00-1.71)$ & $0.050^{*}$ & $40-79$ & [29] \\
\hline Anthocyanidins & & & Italian & $\mathrm{HB}$ & $1294 / 1451$ & & $1.18(0.91-1.53)$ & 0.22 & $46-74$ & [21] \\
\hline
\end{tabular}

Note: a CAPS - The Cancer Prostate Sweden Study; EPIC - The European Prospective Investigation into Cancer and Nutrition; PCANDIET - The Prostate Cancer and Diet Study; WNYDS - The Western New York Diet Study.

${ }^{\mathrm{b}} \mathrm{HB}$ - hospital-based; $\mathrm{PB}$ - population-based.

${ }^{\mathrm{C}} \mathrm{Q} 4$ - quartiles; $\mathrm{Q} 5$ - quintiles.

${ }^{\mathrm{d}} \mathrm{OR}$ - odds ratio; RR - relative risk.

eStatistically significant effects ( $p$ for trend $<0.05$ ) are marked by asterisk.

of both genistein and daidzein against prostate cancer. At that, Nagata et al. described a significant $42 \%$ and $45 \%$ reduction of prostate cancer risk with high dietary consumption of genistein and daidzein, respectively [1], and Lee et al. reported a $47 \%$ and $44 \%$ decrease in the tumor risk with high consumption of these isoflavones, respectively [15]. These results demonstrate that isoflavones can indeed contribute to a protective effect against prostate neoplasms. Also, Strom etal. observed some suggestive, still non-significant, lowering in prostate cancer incidence with high dietary consumption of daidzein and genistein among American men [27]. As the daily intake of soy foods in European countries is very low compared to Asian men (this difference can be even about hundredfold), it is possible that these levels were too low for a preventive effect against prostate cancer to be become obvious [12, 21] (see Table 1).

\section{Prospective cohort studies}

Using the prospective cohort study design, no significant inverse associations with prostate cancer risk were found for intake of total flavonoids as well as flavonoid subclasses flavones, flavanones and anthocyanidins in different populations, including American, Hawaiian, Dutch, and Finnish men [23, 30-33]. Although Wang et al. observed a weak positive relationship with intake of total flavonoids, flavanols or isoflavones, but also flavonols or flavanones with overall prostate cancer incidence, the relative risk varied over follow-up time and the positive association disappeared when the cases diagnosed during the first two follow-up years were excluded from the analysis [16]. The authors proposed that such pattern could be affected by behaviors associated with prostate cancer screening [16]. For flavonols, Knekt et al. found a statistically significant $57 \%$ decrease in prostate cancer risk with high dietary intake of myricetin among Finnish men; but no risk modification by other common flavonols like kaempferol or quercetin [31]. Geybels et al. conducted a prospective cohort study with Dutch men and stratification of the analysis by cancer stage revealed a significant inverse association between intake of both kaempferol and myricetin (not quercetin) with stage IV prostate cancer and not with overall or non-advanced neoplasms (stage I/II tumors) [33]. Similar trend became evident also for total flavanols and epicatechin (not catechin), as the significant inverse association was found for advanced stage prostate tumor and not non-advanced cancer [33].

Although both Park et al. and Kurahashi et al. reported no significant relationships between intake of individual isoflavones (genistein, daidzein) and total prostate cancer risk [3, 23], Kurahashi et al. still found 
Table 2. Epidemiological prospective cohort studies on dietary intake of flavonoids and prostate cancer risk

\begin{tabular}{|c|c|c|c|c|c|c|c|c|c|c|}
\hline $\begin{array}{l}\text { Flavonoid } \\
\text { subclass }\end{array}$ & $\begin{array}{c}\text { Certain } \\
\text { compound }\end{array}$ & Study ${ }^{a}$ & $\begin{array}{l}\text { Popula- } \\
\text { tion }\end{array}$ & $\begin{array}{l}\text { Medi- } \\
\text { an fol- } \\
\text { low-up } \\
\text { (years) }\end{array}$ & $\begin{array}{l}\text { Cases/ } \\
\text { cohort }\end{array}$ & $\begin{array}{l}\text { Intake comparison } \\
\text { (low vs high, } \\
\text { mg/day })^{\mathrm{b}}\end{array}$ & $\begin{array}{c}\text { Multivariate- } \\
\text { adjusted } \mathrm{RR} / \mathrm{HR}^{\mathrm{c}}\end{array}$ & $\begin{array}{l}p \text { for } \\
\text { trend }^{d}\end{array}$ & Comments ${ }^{\mathrm{e}}$ & $\begin{array}{l}\text { Refe- } \\
\text { rence }\end{array}$ \\
\hline Flavonoids & & CPS-II & American & 7.8 & $\begin{array}{c}3974 / \\
43,268\end{array}$ & $\begin{array}{c}<126.6 v s \\
\geqslant 359.5(Q 5)\end{array}$ & $1.11(1.01-1.23)$ & $0.02^{*}$ & $\begin{array}{l}50-74 \text { y; positive associ- } \\
\text { ation for high grade can- } \\
\text { cer within the first } 2 \text { y of fol- } \\
\text { low-up, suggestive inverse } \\
\text { association after excluding } \\
\text { these first } 2 \text { y; no associa- } \\
\text { tions with advanced cancer }\end{array}$ & [16] \\
\hline Flavonoids & & MEC & $\begin{array}{l}\text { American, } \\
\text { Hawaiian }\end{array}$ & 8.0 & $\begin{array}{c}4404 / \\
82,483\end{array}$ & $\begin{array}{l}<1.6 \mathrm{vs} \geqslant 7.2 / \\
1000 \mathrm{kcal}(Q 5)\end{array}$ & $0.93(0.83-1.04)$ & 0.17 & $\begin{array}{l}45-75 \text { y; multiethnic; no in- } \\
\text { verse association regard- } \\
\text { less of stage }\end{array}$ & [23] \\
\hline Flavonoids & & NLCS & Dutch & 17.3 & $\begin{array}{c}3362 / \\
58,279\end{array}$ & 13.1 vs $40.6(\mathrm{Q} 4)$ & $1.00(0.84-1.18)$ & 0.74 & $\begin{array}{l}\text { 55- } 69 \text { y; no significant ef- } \\
\text { fect modification by can- } \\
\text { cer stage }\end{array}$ & [33] \\
\hline Flavonoids & & FMC & Finnish & 24.0 & $62 / 9959$ & $<2.1 v s>4.8(\mathrm{Q} 4)$ & $1.39(0.56-3.46)$ & & $15-99$ y & [30] \\
\hline Flavonoids & & FMC & Finnish & 30.0 & $95 / 5218$ & 4.3 vs 26.9 (Q4) & $1.11(0.61-2.01)$ & 0.57 & & [31] \\
\hline Flavonoids & & KIHD & Finnish & 16.2 & $138 / 2590$ & 9.1 vs 416.3 (Q4) & $1.16(0.58-2.34)$ & 0.831 & $42-60 y$ & [32] \\
\hline Flavones & & CPS-II & American & 7.8 & $\begin{array}{c}3974 / \\
43,268\end{array}$ & $\begin{array}{l}<0.5 v s \\
\geqslant 2.2(Q 5)\end{array}$ & $1.05(0.95-1.16)$ & 0.19 & $\begin{array}{l}50-74 \text { y; no associations for } \\
\text { high grade or advanced can- } \\
\text { cers within or without the first } \\
2 \text { y of follow-up }\end{array}$ & [16] \\
\hline Flavones & & KIHD & Finnish & 16.2 & $138 / 2590$ & (Q4) & $0.71(0.30-1.65)$ & 0.561 & $42-60 y$ & [32] \\
\hline Flavonols & & CPS-II & American & 7.8 & $\begin{array}{c}3974 / \\
43,268\end{array}$ & $\begin{aligned} & <8.9 v S \\
\geqslant & 20.5(Q 5)\end{aligned}$ & $1.10(0.99-1.21)$ & $0.05^{\star}$ & $\begin{array}{l}50-74 \text { y; suggestive pos- } \\
\text { itive association for high } \\
\text { grade cancer within the first } \\
2 \text { y of follow-up, not after } \\
\text { excluding these first } 2 \text { y; } \\
\text { no associations with ad- } \\
\text { vanced cancer }\end{array}$ & [16] \\
\hline Flavonols & & KIHD & Finnish & 16.2 & $138 / 2590$ & (Q4) & $1.53(0.72-3.23)$ & 0.585 & $42-60$ y & [32] \\
\hline Flavonols & Kaempferol & NLCS & Dutch & 17.3 & $\begin{array}{c}3362 / \\
58,279\end{array}$ & 2.5 vs $11.2(\mathrm{Q} 4)$ & $0.98(0.83-1.16)$ & 0.73 & $\begin{array}{l}55-69 \mathrm{y} \text {; significant inverse } \\
\text { association for stage IV can- } \\
\text { cer }[0.78(0.61-1.00) \text {, } \\
\left.p=0.03^{\star}\right] \text {, not for non-ad- } \\
\text { vanced cancer }\end{array}$ & [33] \\
\hline Flavonols & Kaempferol & FMC & Finnish & 30.0 & $95 / 5218$ & 0.1 vs 0.8 (Q4) & $1.03(0.53-2.02)$ & 0.54 & & [31] \\
\hline Flavonols & Myricetin & NLCS & Dutch & 17.3 & $\begin{array}{c}3362 / \\
58,279\end{array}$ & 0.4 vs 2.6 (Q4) & $0.89(0.75-1.05)$ & 0.41 & $\begin{array}{l}55-69 \text { y; signifi- } \\
\text { cant inverse associa- } \\
\text { tion for stage IV can- } \\
\text { cer }[0.71(0.55-0.91) \\
\left.p=0.03^{\star}\right], \text { not for non-ad- } \\
\text { vanced cancer }\end{array}$ & [33] \\
\hline Flavonols & Myricetin & FMC & Finnish & 30.0 & $95 / 5218$ & 0 vs 0.11 (Q4) & $0.43(0.22-0.86)$ & $0.002^{\star}$ & & [31] \\
\hline Flavonols & Quercetin & NLCS & Dutch & 17.3 & $\begin{array}{c}3362 / \\
58,279\end{array}$ & 8.8 vs 28.5 (Q4) & $1.01(0.85-1.19)$ & 0.98 & $\begin{array}{l}55-69 \text { y; no significant ef- } \\
\text { fect modification by can- } \\
\text { cer stage }\end{array}$ & [33] \\
\hline Flavonols & Quercetin & FMC & Finnish & 30.0 & $95 / 5218$ & 1.5 vs 3.9 (Q4) & $0.76(0.40-1.42)$ & 0.35 & & [31] \\
\hline Flavanones & & CPS-II & American & 7.8 & $\begin{array}{c}3974 / \\
43,268\end{array}$ & $\begin{aligned} & <7.5 v s \\
\geqslant & 38.2(Q 5)\end{aligned}$ & $1.08(0.98-1.20)$ & 0.31 & $\begin{array}{l}50-74 \text { y; positive associ- } \\
\text { ation for high grade can- } \\
\text { cer within the first } 2 \text { y of fol- } \\
\text { low-up, not after excluding } \\
\text { these first } 2 \mathrm{y} \text {; no associa- } \\
\text { tions with advanced cancer }\end{array}$ & {$[16]$} \\
\hline Flavanones & & KIHD & Finnish & 16.2 & $138 / 2590$ & (Q4) & $0.90(0.37-2.20)$ & 0.518 & $42-60$ y & [32] \\
\hline Flavanones & Hesperetin & FMC & Finnish & 30.0 & $95 / 5218$ & 0 vs 15.4 (Q4) & $1.47(0.80-2.71)$ & 0.26 & & {$[31]$} \\
\hline Flavanones & Naringenin & FMC & Finnish & 30.0 & $95 / 5218$ & 0 vs 4.7 (Q4) & $1.48(0.80-2.73)$ & 0.27 & & [31] \\
\hline Flavanols & & CPS-II & American & 7.8 & $\begin{array}{c}3974 / \\
43,268\end{array}$ & $\begin{array}{c}<10.4 \text { vs } \\
\geqslant 37.9(\mathrm{Q} 5)\end{array}$ & $1.18(1.06-1.30)$ & $0.01^{*}$ & $\begin{array}{l}50-74 \text { y; suggestive pos- } \\
\text { itive association for high } \\
\text { grade cancer within the first } \\
2 \text { y of follow-up, not after } \\
\text { excluding these first } 2 \text { y; } \\
\text { no associations with ad- } \\
\text { vanced cancer }\end{array}$ & {$[16]$} \\
\hline Flavanols & & NLCS & Dutch & 17.3 & $\begin{array}{c}3362 / \\
58,279\end{array}$ & 14.5 vs 98.7 (Q4) & $0.97(0.82-1.15)$ & 0.58 & $\begin{array}{l}55-69 \text { y; signifi- } \\
\text { cant inverse associa- } \\
\text { tion for stage IV can- } \\
\text { cer }[0.73(0.57-0.95) \\
\left.p=0.01^{\star}\right], \text { not for non-ad- } \\
\text { vanced cancer }\end{array}$ & [33] \\
\hline Flavanols & & KIHD & Finnish & 16.2 & $138 / 2590$ & (Q4) & $1.37(0.65-2.89)$ & 0.82 & $42-60$ y & [32] \\
\hline Flavanols & Catechin & NLCS & Dutch & 17.3 & $\begin{array}{c}3362 / \\
58,279\end{array}$ & 1.9 vs $6.8(\mathrm{Q} 4)$ & $1.03(0.88-1.22)$ & 0.83 & $\begin{array}{l}\text { 55- } 69 \text { y; no significant ef- } \\
\text { fect modification by can- } \\
\text { cer stage }\end{array}$ & [33] \\
\hline
\end{tabular}




\begin{tabular}{|c|c|c|c|c|c|c|c|c|c|c|}
\hline $\begin{array}{l}\text { Flavonoid } \\
\text { subclass }\end{array}$ & $\begin{array}{c}\text { Certain } \\
\text { compound }\end{array}$ & Study ${ }^{a}$ & $\begin{array}{l}\text { Popula- } \\
\text { tion }\end{array}$ & $\begin{array}{l}\text { Medi- } \\
\text { an fol- } \\
\text { low-up } \\
\text { (years) }\end{array}$ & $\begin{array}{l}\text { Cases/ } \\
\text { cohort }\end{array}$ & $\begin{array}{l}\text { Intake comparison } \\
\text { (low vs high, } \\
\text { mg/day })^{\mathrm{b}}\end{array}$ & $\begin{array}{c}\text { Multivariate- } \\
\text { adjusted RR/HR }\end{array}$ & $\begin{array}{l}p \text { for } \\
\text { trend }^{d}\end{array}$ & Comments $^{\mathrm{e}}$ & $\begin{array}{l}\text { Refe- } \\
\text { rence }\end{array}$ \\
\hline Flavanols & Epicatechin & NLCS & Dutch & 17.3 & $\begin{array}{c}3362 / \\
58,279\end{array}$ & 5.3 vs 20.8 (Q4) & $1.01(0.86-1.20)$ & 0.62 & $\begin{array}{l}55-69 \mathrm{y} ; \text { significant in- } \\
\text { verse association for stage } \\
\text { III/IV }[0.84(0.68-1.04), \\
\left.p=0.05^{\star}\right] \text { and IV can- } \\
\text { cer }[0.74(0.57-0.95), \\
\left.p=0.01^{\star}\right], \text { not for non-ad- } \\
\text { vanced cancer }\end{array}$ & [33] \\
\hline Isoflavones & & CPS-II & American & 7.8 & $\begin{array}{c}3974 / \\
43,268\end{array}$ & $\begin{array}{c}<0.029 v s \\
\geqslant 0.144(Q 5)\end{array}$ & $1.11(1.01-1.22)$ & $<0.001^{*}$ & $\begin{array}{l}50-74 \text { y; no associations } \\
\text { for high grade or advanced } \\
\text { cancers within or without } \\
\text { the first } 2 \text { y of follow-up }\end{array}$ & [16] \\
\hline Isoflavones & Genistein & MEC & $\begin{array}{l}\text { American, } \\
\text { Hawaiian }\end{array}$ & 8.0 & $\begin{array}{c}4404 / \\
82,483\end{array}$ & $\begin{array}{l}<0.7 \text { vs } \geqslant 3.1 / \\
1000 \mathrm{kcal}(Q 5)\end{array}$ & $0.94(0.84-1.04)$ & 0.16 & $\begin{array}{l}45-75 \text { y; multiethnic; no in- } \\
\text { verse association regard- } \\
\text { less of stage }\end{array}$ & [23] \\
\hline Isoflavones & Genistein & JPHC & Japanese & 5.0 & $\begin{array}{c}307 / \\
43,509\end{array}$ & $\begin{array}{c}<13.2 v s \\
\geqslant 32.8(\mathrm{Q} 4)\end{array}$ & $0.71(0.48-1.03)$ & 0.22 & $\begin{array}{l}45-74 \text { y; non-significant in- } \\
\text { verse association for lo- } \\
\text { calized, not advanced } \\
\text { cancer; significant in- } \\
\text { verse association for men } \\
>60 \text { y with localized can- } \\
\text { cer }[0.52(0.30-0.90) \text {, } \\
\left.p=0.03^{\star}\right], \text { not advanced } \\
\text { cancer or for younger men }\end{array}$ & [3] \\
\hline Isoflavones & Daidzein & MEC & $\begin{array}{l}\text { American, } \\
\text { Hawaiian }\end{array}$ & 8.0 & $\begin{array}{c}4404 / \\
82,483\end{array}$ & $\begin{array}{c}<0.7 \text { vs } \\
\geqslant 3.2 / 1000 \mathrm{kcal} \\
(\mathrm{Q} 5)\end{array}$ & $0.92(0.82-1.02)$ & 0.09 & $\begin{array}{l}45-75 \text { y; multiethnic; no in- } \\
\text { verse association regard- } \\
\text { less of stage }\end{array}$ & [23] \\
\hline Isoflavones & Daidzein & JPHC & Japanese & 5.0 & $\begin{array}{c}307 / \\
43,509\end{array}$ & $\begin{aligned} & <8.5 v s \\
\geqslant & 20.4(Q 4)\end{aligned}$ & $0.77(0.52-1.13)$ & 0.43 & $\begin{array}{l}45-74 \text { y; non-significant in- } \\
\text { verse association for lo- } \\
\text { calized, not advanced } \\
\text { cancer; significant in- } \\
\text { verse association for men } \\
>60 \text { y with localized can- } \\
\text { cer }[0.50(0.28-0.88) \text {, } \\
\left.p=0.04^{\star}\right], \text { not advanced } \\
\text { cancer or for younger men }\end{array}$ & [3] \\
\hline Isoflavones & Glycitein & MEC & $\begin{array}{l}\text { American, } \\
\text { Hawaiian }\end{array}$ & 8.0 & $\begin{array}{c}4404 / \\
82,483\end{array}$ & $\begin{array}{c}<0.18 v s \\
\geqslant 0.80 / 1000 \mathrm{kcal} \\
(\mathrm{Q} 5)\end{array}$ & $0.91(0.82-1.01)$ & 0.07 & $\begin{array}{l}45-75 \text { y; multiethnic; no in- } \\
\text { verse association regard- } \\
\text { less of stage }\end{array}$ & [23] \\
\hline Anthocyanidins & & CPS-II & American & 7.8 & $\begin{array}{c}3974 / \\
43,268\end{array}$ & $\begin{aligned} & <5.9 v s \\
\geqslant & 18.0(\mathrm{Q} 5)\end{aligned}$ & $1.10(1.00-1.22)$ & 0.13 & $\begin{array}{l}50-74 \text { y; no associations } \\
\text { for high grade or advanced } \\
\text { cancers within or without } \\
\text { the first } 2 \text { y of follow-up }\end{array}$ & {$[16]$} \\
\hline Anthocyanidins & & KIHD & Finnish & 16.2 & $138 / 2590$ & (Q4) & $0.59(0.24-1.41)$ & 0.974 & $42-60$ y & [32] \\
\hline
\end{tabular}

Note: ${ }^{a}$ CPS-II - The Cancer Prevention Study II Nutrition Cohort; FMC - The Finnish Mobile Clinic Health Examination Survey; JPHC - The Japan Public Health Center-based prospective study; KIHD - The Kuopio Ischaemic Heart Disease Risk Factor Study; MEC - The Multiethnic Cohort Study; NLCS - The Netherlands Cohort Study.

${ }^{\mathrm{b}} \mathrm{Q} 4$ - quartiles; Q5 - quintiles.

${ }^{\mathrm{c}} \mathrm{RR}$ - relative risk; HR - hazard ratio.

dStatistically significant effects ( $p$ for trend $<0.05$ ) are marked by asterisk. ${ }^{\text {e}}$ Age in baseline.

an inverse association between intake of both genistein and daidzein and localized cancer [3]. In particular, Japanese men older than 60 years had about $50 \%$ lower risk of localized prostate cancer with high dietary consumption of genistein and daidzein [3] (see Table 2). Thus, compared to findings from case-control studies, the evidence from prospective cohort studies about the protective effects of flavonoids against prostate cancer is much more sparse being restricted to certain subgroups (stratified by age of subjects or cancer stage).

\section{Biomarker studies}

Unlike the works assessing retrospectively or prospectively dietary intake of different flavonoids, biomarker studies have focused only to isoflavones and their metabolites. At that, no associations were observed between total serum or urinary isoflavones and prostate cancer risk in English [34] or Scottish men [5].
Null associations were detected also for individual serum isoflavones and their urinary biomarkers (genistein, daidzein, glycitein, O-desmethylangolensin, equol) within English men [12, 34] and for serum isoflavones among older Scottish men [5]. In a large prospective investigation performed with men from eight European countries, no significant relationship was reported for plasma genistein, daidzein or equol and prostate cancer risk [24, 35]. In the work conducted with Jamaican men, no association was found between urinary excretion of genistein or daidzein and prostate cancer incidence, but a significant inverse relationship was found for urinary equol concentration when non-producers of equol were assessed as reference group [36]. This study indicated that men who were able to produce equol from daidzein by the aid of specific intestinal microflora were at reduced risk of total and high grade (but not low 
Table 3. Epidemiological studies on biomarkers of flavonoids and prostate cancer risk

\begin{tabular}{|c|c|c|c|c|c|c|c|c|c|}
\hline $\begin{array}{l}\text { Flavonoid } \\
\text { subclass }\end{array}$ & $\begin{array}{c}\text { Certain } \\
\text { compound }\end{array}$ & $\begin{array}{l}\text { Bio- } \\
\text { marker }\end{array}$ & Study ${ }^{a}$ & $\begin{array}{l}\text { Popula- } \\
\text { tion }\end{array}$ & $\begin{array}{l}\text { Cases/ } \\
\text { controls }\end{array}$ & $\begin{array}{c}\text { Multivariate- } \\
\text { adjusted OR/RR }\end{array}$ & $\begin{array}{c}p \text { for } \\
\text { trend }\end{array}$ & Comments $^{\mathrm{d}}$ & $\begin{array}{l}\text { Refe- } \\
\text { rence }\end{array}$ \\
\hline Isoflavones & & Serum & EPIC-Norfolk & English & $191 / 815$ & $1.01(0.93-1.10)$ & 0.809 & $45-75$ y & [34] \\
\hline Isoflavones & & Serum & PCANDIET & Scottish & $238 / 198$ & $1.24(0.69-2.20)$ & 0.64 & $50-74$ y & [5] \\
\hline Isoflavones & & Urinary & EPIC-Norfolk & English & $152 / 665$ & $0.98(0.90-1.08)$ & 0.727 & $45-75 y$ & [34] \\
\hline Isoflavones & Genistein & Plasma & EPIC & European & $1605 / 1697$ & $1.00(0.79-1.27)$ & 0.82 & $\begin{array}{l}43-76 y \text {; from eight countries; no associations } \\
\text { as stratified by cancer stage (localized or ad- } \\
\text { vanced) or histological grade (low or high), } \\
\text { or age of men at diagnosis ( }<60 \mathrm{y} \text { or } \geqslant 60 \mathrm{y} \text { ) }\end{array}$ & [35] \\
\hline Isoflavones & Genistein & Plasma & EPIC & European & $950 / 1042$ & $0.74(0.54-1.00)$ & $0.051^{*}$ & $43-76$ y; from eight countries & [24] \\
\hline Isoflavones & Genistein & Plasma & JPHC & Japanese & $201 / 402$ & $0.66(0.40-1.08)$ & 0.08 & $\begin{array}{l}40-69 \mathrm{y} \text {; significant inverse association } \\
\text { for localized cancer }[0.54(0.29-1.01) \text {, } \\
\left.p=0.03^{\star}\right] \text {, not advanced cancer }\end{array}$ & {$[5]$} \\
\hline Isoflavones & Genistein & Serum & JACC & Japanese & $52 / 151$ & $0.76(0.32-1.82)$ & 0.54 & $\begin{array}{l}\geqslant 40 \mathrm{y} ; \text { suggestive inverse association for } \\
\text { men with } \mathrm{T} \geqslant 6.9 \mathrm{nM} \text {, or } \mathrm{T} \geqslant 6.9 \mathrm{nM} \text { and PSA } \\
\leqslant 10 \mathrm{ng} / \mathrm{ml} \text { for cases, }<4 \mathrm{ng} / \mathrm{ml} \text { for controls }\end{array}$ & [25] \\
\hline Isoflavones & Genistein & Plasma & & Chinese & $46 / 54$ & $0.31(0.13-0.71)$ & $0.006^{*}$ & & [4] \\
\hline Isoflavones & Genistein & Serum & EPIC-Norfolk & English & 191/815 & $0.99(0.93-1.05)$ & 0.78 & $45-75$ y & [34] \\
\hline Isoflavones & Genistein & Serum & EPIC-Norfolk & English & $89 / 178$ & $1.01(0.83-1.23)$ & 0.938 & $45-75 y$ & [12] \\
\hline Isoflavones & Genistein & Serum & PCANDIET & Scottish & $238 / 198$ & $1.36(0.76-2.43)$ & 0.37 & $50-74$ y & [5] \\
\hline Isoflavones & Genistein & Urinary & MEC & $\begin{array}{l}\text { American, } \\
\text { Hawaiian }\end{array}$ & $249 / 404$ & $0.72(0.40-1.31)$ & 0.09 & $\begin{array}{l}\text { 45-75 y; multiethnic; suggestive inverse } \\
\text { association for localized and advanced can- } \\
\text { cer, and for Latinos and Whites (not Afri- } \\
\text { can Americans and Japanese Americans) }\end{array}$ & [37] \\
\hline Isoflavones & Genistein & Urinary & & Jamaican & $175 / 194$ & $1.23(0.67-2.56)$ & 0.502 & $\begin{array}{l}40-80 \text { y; no associations as stratified } \\
\text { by cancer grades (low or high) }\end{array}$ & [36] \\
\hline Isoflavones & Genistein & Urinary & EPIC-Norfolk & English & $152 / 665$ & $1.00(0.95-1.05)$ & 0.86 & $45-75$ y & [34] \\
\hline Isoflavones & Genistein & Urinary & EPIC-Norfolk & English & $89 / 178$ & $0.92(0.76-1.12)$ & 0.415 & $45-75$ y & [12] \\
\hline Isoflavones & Daidzein & Plasma & EPIC & European & $950 / 1042$ & $0.80(0.60-1.07)$ & 0.209 & $43-76$ y; from eight countries & [24] \\
\hline Isoflavones & Daidzein & Plasma & JPHC & Japanese & $201 / 402$ & $0.78(0.49-1.25)$ & 0.44 & $\begin{array}{l}\text { 40-69 y; suggestive inverse association } \\
\text { for localized, not advanced cancer }\end{array}$ & [6] \\
\hline Isoflavones & Daidzein & Serum & EPIC-Norfolk & English & $89 / 178$ & $1.01(0.86-1.18)$ & 0.902 & $45-75$ y & [12] \\
\hline Isoflavones & Daidzein & Serum & EPIC-Norfolk & English & 191/815 & $0.99(0.93-1.05)$ & 0.68 & $45-75 y$ & [34] \\
\hline Isoflavones & Daidzein & Serum & PCANDIET & Scottish & $247 / 200$ & $1.34(0.76-2.38)$ & 0.21 & $50-74 y$ & {$[5]$} \\
\hline Isoflavones & Daidzein & Serum & JACC & Japanese & $52 / 151$ & $0.74(0.31-1.76)$ & 0.50 & $\begin{array}{l}\geqslant 40 \mathrm{y} \text {; suggestive inverse association for } \\
\text { men with } \mathrm{T} \geqslant 6.9 \mathrm{nM} \text {, or } \mathrm{T} \geqslant 6.9 \mathrm{nM} \text { and PSA } \\
\leqslant 10 \mathrm{ng} / \mathrm{ml} \text { for cases, }<4 \mathrm{ng} / \mathrm{ml} \text { for controls }\end{array}$ & {$[25]$} \\
\hline Isoflavones & Daidzein & Urinary & MEC & $\begin{array}{l}\text { American, } \\
\text { Hawaiian }\end{array}$ & $249 / 404$ & $0.55(0.31-0.98)$ & $0.03^{*}$ & $\begin{array}{l}45-75 \text { y; multiethnic; suggestive inverse } \\
\text { associations for localized and advanced } \\
\text { cancer, and for different ethnic groups (Af- } \\
\text { rican Americans, Japanese Americans, La- } \\
\text { tinos and Whites) }\end{array}$ & [37] \\
\hline Isoflavones & Daidzein & Urinary & & Jamaican & $175 / 194$ & $0.85(0.47-1.54)$ & 0.600 & $\begin{array}{l}40-80 \text { y; no associations as stratified } \\
\text { by cancer grades (low or high) }\end{array}$ & {$[36]$} \\
\hline Isoflavones & Daidzein & Urinary & EPIC-Norfolk & English & $152 / 665$ & $0.97(0.92-1.03)$ & 0.31 & $45-75$ y & [34] \\
\hline Isoflavones & Daidzein & Urinary & EPIC-Norfolk & English & $89 / 178$ & $0.99(0.83-1.17)$ & 0.866 & $45-75 y$ & [12] \\
\hline Isoflavones & Glycitein & Plasma & JPHC & Japanese & $201 / 402$ & $0.78(0.48-1.26)$ & 0.51 & $\begin{array}{l}40-69 \text { y; no associations as stratified } \\
\text { by cancer stage (localized or advanced) }\end{array}$ & {$[6]$} \\
\hline Isoflavones & Glycitein & Serum & EPIC-Norfolk & English & $191 / 815$ & $1.01(0.95-1.08)$ & 0.67 & $45-75$ y & [34] \\
\hline Isoflavones & Glycitein & Serum & EPIC-Norfolk & English & $89 / 178$ & $1.08(0.49-2.38)$ & 0.848 & $45-75$ y & [12] \\
\hline Isoflavones & Glycitein & Urinary & EPIC-Norfolk & English & $152 / 665$ & $1.01(0.96-1.06)$ & 0.81 & $45-75$ y & [34] \\
\hline Isoflavones & Glycitein & Urinary & EPIC-Norfolk & English & $89 / 178$ & $1.01(0.82-1.26)$ & 0.901 & $45-75 y$ & [12] \\
\hline Isoflavones & $\begin{array}{l}\text { O-Des- } \\
\text { methylan- } \\
\text { golensin }\end{array}$ & Serum & EPIC-Norfolk & English & $191 / 815$ & $0.97(0.91-1.03)$ & 0.33 & $45-75$ y & {$[34]$} \\
\hline Isoflavones & $\begin{array}{l}\text { O-Des- } \\
\text { methylan- } \\
\text { golensin }\end{array}$ & Serum & EPIC-Norfolk & English & $89 / 178$ & $0.68(0.36-1.29)$ & 0.236 & $45-75$ y & {$[12]$} \\
\hline Isoflavones & $\begin{array}{l}\text { O-Des- } \\
\text { methylan- } \\
\text { golensin }\end{array}$ & Urinary & EPIC-Norfolk & English & $152 / 665$ & $0.98(0.94-1.03)$ & 0.42 & $45-75 y$ & [34] \\
\hline Isoflavones & $\begin{array}{l}\text { O-Des- } \\
\text { methylan- } \\
\text { golensin }\end{array}$ & Urinary & EPIC-Norfolk & English & $89 / 178$ & $0.93(0.73-1.18)$ & 0.540 & $45-75 y$ & [12] \\
\hline Isoflavones & Equol & Plasma & EPIC & European & $950 / 1042$ & $0.99(0.70-1.39)$ & 0.461 & $43-76$ y; from eight countries & {$[24]$} \\
\hline Isoflavones & Equol & Plasma & JPHC & Japanese & $201 / 402$ & $0.60(0.36-0.99)$ & $0.04^{\star}$ & $\begin{array}{l}40-69 \mathrm{y} \text {; significant inverse association } \\
\text { for localized cancer }[0.43(0.22-0.82) \text {, } \\
\left.p=0.02^{*}\right] \text {, not advanced cancer }\end{array}$ & [6] \\
\hline Isoflavones & Equol & Serum & EPIC-Norfolk & English & $191 / 815$ & $1.02(0.96-1.08)$ & 0.52 & $45-75 y$ & [34] \\
\hline Isoflavones & Equol & Serum & EPIC-Norfolk & English & $89 / 178$ & $1.32(0.73-2.38)$ & 0.358 & $45-75 y$ & [12] \\
\hline Isoflavones & Equol & Serum & PCANDIET & Scottish & $247 / 200$ & $1.07(0.71-1.61)$ & 0.75 & $50-74 y$ & [5] \\
\hline Isoflavones & Equol & Serum & JACC & Japanese & $52 / 151$ & $0.39(0.15-0.98)$ & $0.046^{\star}$ & $\begin{array}{l}\geqslant 40 \mathrm{y} \text {; suggestive inverse association for } \\
\text { men with } T \geqslant 6.9 \mathrm{nM} \text {, or } T \geqslant 6.9 \mathrm{nM} \text { and PSA }\end{array}$ & [25] \\
\hline Isoflavones & Equol & Urinary & MEC & $\begin{array}{l}\text { American, } \\
\text { Hawaiian }\end{array}$ & $249 / 404$ & $1.32(0.84-2.08)$ & 0.08 & $\begin{array}{l}\leqslant 10 \mathrm{ng} / \mathrm{ml} \text { for cases, }<4 \mathrm{ng} / \mathrm{ml} \text { for controls } \\
45-75 \mathrm{y} ; \text { multiethnic; no associations } \\
\text { for localized and advanced cancer or dif- } \\
\text { ferent ethnic groups (African Americans, } \\
\text { Japanese Americans, Latinos and Whites) }\end{array}$ & [37] \\
\hline
\end{tabular}




\begin{tabular}{|c|c|c|c|c|c|c|c|c|c|}
\hline $\begin{array}{c}\text { Flavonoid } \\
\text { subclass }\end{array}$ & $\begin{array}{c}\text { Certain } \\
\text { compound }\end{array}$ & $\begin{array}{c}\text { Bio- } \\
\text { marker }\end{array}$ & Study ${ }^{a}$ & $\begin{array}{l}\text { Popula- } \\
\text { tion }\end{array}$ & $\begin{array}{l}\text { Cases/ } \\
\text { controls }\end{array}$ & $\begin{array}{c}\text { Multivariate- } \\
\text { adjusted OR/RR }\end{array}$ & $\begin{array}{l}p \text { for } \\
\text { trend }\end{array}$ & Comments $^{d}$ & $\begin{array}{l}\text { Refe- } \\
\text { rence }\end{array}$ \\
\hline Isoflavones & Equol & Urinary & & Jamaican & $175 / 194$ & $0.48(0.26-0.87)$ & $0.020^{\star}$ & $\begin{array}{l}40-80 \mathrm{y} \text {; significant inverse association } \\
\text { for high grade cancer }[0.29(0.13-0.60) \text {, } \\
\left.p=0.001^{\star}\right] \text {, not low grade cancer }\end{array}$ & {$[36]$} \\
\hline Isoflavones & Equol & Urinary & EPIC-Norfolk & English & $152 / 665$ & $1.03(0.97-1.07)$ & 0.20 & $45-75$ y & [34] \\
\hline Isoflavones & Equol & Urinary & EPIC-Norfolk & English & $89 / 178$ & $1.21(0.93-1.57)$ & 0.147 & $45-75 y$ & [12] \\
\hline
\end{tabular}

Note: aEPIC - The European Prospective Investigation into Cancer and Nutrition; JACC - The Japan Collaborative Cohort Study; JPHC - The Japan Public Health Center-based Prospective Study; MEC - The Multiethnic Cohort Study; PCANDIET - The Prostate Cancer and Diet Study.

${ }^{\mathrm{b}} \mathrm{OR}$ - odds ratio; $\mathrm{RR}$ - relative risk; the risk for equol is presented in comparison to equol non-producers.

'Statistically significant effects $(p$ for trend $<0.05)$ are marked by asterisk.

${ }^{\mathrm{d} P S A}$ - prostate-specific antigen; $\mathrm{T}$ - testosterone.

grade) prostate cancer as compared to non-producers of this metabolite [36]. However, another study that measured urinary biomarkers of isoflavones did not observe such protective effect for equol [37]. Quite contrary, urinary excretion of daidzein was significantly inversely associated with prostate cancer risk among multiethnic men living in Hawaii and Los Angeles and a respective suggestive trend was indicated also for genistein, regardless of the stage of tumor [37].

Differently from works performed with European and American men, their Asian counterparts consume substantially higher amounts of soy foods and consequently also isoflavones. Wu etal. reported a $69 \%$ reduced prostate cancer risk among Chinese men consuming high amounts of genistein as evaluated by plasma genistein level [4]. Kurahashi et al. described a significant $44 \%$ reduction in the risk of localized (not advanced) prostate tumor among Japanese men with higher plasma genistein concentrations and a similar suggestive tendency was reported also for plasma daidzein level [6]. Plasma equol level was inversely related to both overall as well as localized prostate cancer incidence, not modifying the risk of advanced cancer [6]. A significant $61 \%$ decrease in prostate cancer risk was detected also in another study with Japanese men with high serum equol levels and similar suggestive, still non-significant, trend was observed for genistein and daidzein when the subjects with low serum testosterone levels were excluded [25]. These studies with Asian men support that high doses of isoflavones can be indeed preventive against prostate cancer development (see Table 3).

\section{POTENTIAL FACTORS GIVING RISE TO INCONCISTENCIES}

As come into sight from the epidemiological findings presented in above paragraphs, there are still relatively few data published about the associations between flavonoids and prostate cancer risk, and these results are rather inconclusive. There are several potential reasons providing some explanations to these inconsistencies.

First, assessment of intake of flavonoids has been limited for a long time due to lack of data about food composition. Exposure to flavonoids can be estimated by either food diaries or food frequency questionnaires and these data are further translated to dietary intake using quantitative food composition databases [12]. However, reliable information about the content of flavonoids in different plant-derived food items was not available until the last decade, when several databases were made public for scientific community $[16,21$, 23]. However, these food composition databases are not able to capture the variability of flavonoid content attributable to cultivars, growing methods and environmental conditions, exposure to sunlight and heat, or harvesting time and processing practices [21, 33, 38]. Also, these data tables can not detect all sources of flavonoids in the human diet [37]. Furthermore, some questions arise by adaptation of food composition databases created in the US to the diet consumed in European countries [5, 21].

Second, another possible reason for the inconsistent results of epidemiological studies can come from the differences in level of flavonoids intake. For example, it has been demonstrated that the mean daily consumption of isoflavones is low in Western populations $(<3 \mathrm{mg}$ ), whereas it is considerably higher among Asian subjects reaching even $70-100 \mathrm{mg}$ and being for most Asian men in the range of 30 to $50 \mathrm{mg}[1,4,5,7$, 15-17, 23]. Therefore, it can be that the consumption of isoflavones in Western countries is too low to observe a protective action against prostate tumor [4, $5,9,10,12,16,23,35]$. Whether similar effects exist also for other flavonoids and their subclasses, i.e. the risk-lowering response manifests only at a certain level, is still unknown. The possible age periods of the highest susceptibility to beneficial effects of flavonoids have also remained to be determined. Furthermore, we can not rule out the possibility that follow-up times of prospective cohort studies were not long enough for detection of protective action, especially in the case of advanced neoplasms [16].

Third, it is well known that following to their ingestion, flavonoids undergo an extensive metabolic conversion in the intestine and liver, as a result of which various conjugates with potentially altered biological activity enter bloodstream and reach target tissues, and are finally excreted in urine $[11,12]$. Blood and urinary biomarkers can reflect dietary consumption of flavonoids, capturing intake from all food sources, considering also their metabolic biotransformation [24, 34]. Therefore, direct measurement of these biomarkers might provide a more relevant insight into the associations between exposure to flavonoids and prostate cancer risk than assessment of food consumption [34]. However, besides dietary intake, the levels of flavonoids in biological fluids are affected by intestinal microflora, diet composition and content of fat in the food, consumption of alcohol, and the use of antibiotics as well as possible bowel diseases $[5,28,36]$. Moreover, biomarkers reflect only 
short-term intake of flavonoids due to their brief halflives in the human body and might not accurately reflect the long-term dietary habits; especially, when only one serum measurement or spot urine test is used $[2,5,6$, 35-37]. Thus, estimation of dietary intake has remained indispensable in epidemiological studies, whereas measurement of biomarkers can complement dietary assessment with bioavailability issues. At that, about $30-50 \%$ of people are able to metabolize daidzein to equol, the most biologically active isoflavone, by the aid of special intestinal bacteria and this ability varies within different ethnic populations [ $8,9,19,36,38]$. Moreover, it has been shown that the prevalence of equol-producers is generally lower among subjects suffering from prostate cancer compared to healthy controls [2, 8, 19]. For example, it was shown that just $29 \%$ of Japanese men with prostate cancer could produce equol, compared to $46 \%$ of controls and these estimates were $30 \%$ and $59 \%$ for Korean cases and controls, respectively [8]. If further studies confirm that equol can lower the prostate cancer risk and intestinal environment without equol-converting bacteria is indeed a risk factor for this malignancy, a possible prevention strategy for future would be to improve intestinal microflora by probiotic technology, enabling it to generate equol [7, 9].

In the case of retrospective study design, the recall bias is unavoidable. Patients could recall their food consumption differently than controls and it is also possible that they changed their diet after cancer was diagnosed [15, 21]. However, this misclassification was probably non-differential, as the knowledge about possible role of flavonoids on prostate carcinogenesis was not widespread among general public [15, 28]. Analysis of data collected prospectively might reduce the problems of disease-related influences on dietary intake of flavonoid-rich food items or their biomarker levels [34, 37]. However, the number of cases could not be large enough for acceptably strong statistical power, especially for subgroup analyses stratified by different parameters [19, 33, 37].

Another common concern in epidemiological studies is potential confounding. Despite adjustment for diverse aspects, it is always possible that residual confounders due to uncontrolled or as yet unknown factors might remain $[5,12,15,16,26,27,36]$. For example, most of the studies did not collect information about testing of prostate specific antigen that might be a confounder [16, 33]. Also, only few investigations considered histological grade or tumor stage, although risk factors of prostate cancer can vary within subgroups differentiated by disease aggressiveness or stage [8, 26, 33]. In addition, lifestyle related to consumption of higher doses of flavonoids or some dietary covariates might contribute to the susceptibility to prostate carcinogenesis $[3,5$, $10,16]$. Furthermore, Hedelin et al. indicated that health benefits of isoflavone intake against prostate tumor can be restricted to men carrying specific genetic variant in estrogen receptor $\beta$ gene [28].

\section{CONCLUSIONS AND FURTHER PERSPECTIVES}

High incidence rate, slow progression and long course make prostate cancer a perfect disorder for chemoprevention which onset, development and progression can be influenced by certain dietary constituents [20]. There are several epidemiological findings suggesting that high intake of specific flavonoids can lead to reduced prostate cancer risk. Indeed, consumption of isoflavones at doses characteristic to Asian population may modulate the risk of prostate cancer and therefore, high intake of these phytoestrogens might be an efficient protective means against prostate tumorigenesis among Chinese and Japanese men. The stronger preventive effect of isoflavones on localized tumor compared to advanced neoplasm is in agreement with the expression of estrogen receptor $\beta$ in normal prostate tissue and early stages neoplasm, decline in expression during malignant transformation and complete loss in advanced tumor with higher metastatic potential and mortality; supposing that preventive effects of isoflavones are mediated via this receptor subtype [3, $6,28]$. Based on the autopsy studies the rate of latent tumors is similar in Asian and Western countries and the significantly lower incidence of clinically detectable prostate tumors in Asian men can come from blocking and delaying the latent cancer progression by intake of high amounts of isoflavones [3,6]. An important factor in health benefits of high consumption of isoflavones seems to arise also from the ability to metabolize daidzein to equol by possessing a specific intestinal bacterial combination. Whether ethnic variation in this metabolic conversion may provide some explanation to the differences in prostate cancer incidence remains to be further clarified. On the other hand, some epidemiological studies with Western men have demonstrated the protective action of certain flavonols or flavanols manifesting particularly against advanced, later stage tumor. As a whole, these data indicate that through intake of various plant-derived phytochemicals nature can provide protection and chemoprevention against different stages of prostate cancer.

There is no doubt that large-scale and preferentially prospectively designed studies are needed to further investigate the association between exposure to flavonoids and the risk of prostate cancer. At that, subjects with consumption of wider concentration range of these plant metabolites as well as stratification by different factors, including disease stage, are highly needed. If the protective effects will be confirmed, chemopreventive intervention strategies via modification of dietary habits can be developed to reduce the prostate cancer incidence, especially among at-risk men. Last but not least, although adverse effects of flavonoids on the prostate tissue have not been reported, studies considering safety profile of these phytochemicals are never too much.

\section{CONFLICT OF INTEREST}

None declare. 


\section{REFERENCES}

1. Nagata Y, Sonoda T, Mori M, et al. Dietary isoflavones may protect against prostate cancer in Japanese men. J Nutr 2007; 137: 1974-9.

2. Akaza H, Miyanaga N, Takashima N, et al. Comparisons of percent equol producers between prostate cancer patients and controls: case-controlled studies of isoflavones in Japanese, Korean and American residents. Jpn J Clin Oncol 2004; 34: 86-9.

3. Kurahashi N, Iwasaki M, Sasazuki S, et al. Soy product and isoflavone consumption in relation to prostate cancer in Japanese men. Cancer Epidemiol Biomarkers Prev 2007; 16: $538-45$.

4. Wu Y, Zhang L, Na R, et al. Plasma genistein and risk of prostate cancer in Chinese population. Int Urol Nephrol 2015; 47: 965-70.

5. Heald CL, Ritchie MR, Bolton-Smith C, et al. Phytooestrogens and risk of prostate cancer in Scottish men. Br J Nutr 2007; 98: 388-96.

6. Kurahashi N, Iwasaki M, Inoue M, et al. Plasma isoflavones and subsequent risk of prostate cancer in a nested case-control study: the Japan Public Health Center. J Clin Oncol 2008; 26: 5923-9.

7. Akaza H. Prostate cancer chemoprevention by soy isoflavones: role of intestinal bacteria as the "second human genome". Cancer Sci 2012; 103: 969-75.

8. Vastag B. Soy and prostate cancer study results mixed. J Natl Cancer Inst 2007; 99: 1364-5.

9. Sugiyama Y, Masumori N, Fukuta F, et al. Influence of isoflavone intake and equol-producing intestinal flora on prostate cancer risk. Asian Pac J Cancer Prev 2013; 14: 1-4.

10. Ganry O. Phytoestrogens and prostate cancer risk. Prev Med 2005; 41: 1-6.

11. Nambiar D, Singh RP. Advances in prostate cancer chemoprevention: a translational perspective. Nutr Cancer 2013; 65 (Suppl 1): 12-25.

12. Low YL, Taylor JI, Grace PB, et al. Phytoestrogen exposure, polymorphisms in COMT, CYP19, ESR1, and SHBG genes, and their associations with prostate cancer risk. Nutr Cancer 2006; 56: 31-9.

13. Johnson JJ, Bailey HH, Mukhtar H. Green tea polyphenols for prostate cancer chemoprevention: a translational perspective. Phytomedicine 2010; 17: 3-13.

14. Messina MJ. Emerging evidence on the role of soy in reducing prostate cancer risk. Nutr Rev 2003; 61: 117-31.

15. Lee MM, Gomez SL, Chang JS, et al. Soy and isoflavone consumption in relation to prostate cancer risk in China. Cancer Epidemiol Biomarkers Prev 2003; 12: 665-8.

16. Wang Y, Stevens VL, Shah R, et al. Dietary flavonoid and proanthocyanidin intakes and prostate cancer risk in a prospective cohort of US men. Am J Epidemiol 2014; 179: 974-86.

17. Hedelin M, Klint A, Chang ET, et al. Dietary phytoestrogen, serum enterolactone and risk of prostate cancer: the cancer prostate Sweden study (Sweden). Cancer Causes Control 2006; 17: 169-80.

18. McCann SE, Ambrosone $\mathrm{CB}$, Moysich $\mathrm{KB}$, et al. Intakes of selected nutrients, foods, and phytochemicals and prostate cancer risk in western New York. Nutr Cancer 2005; 53: $33-41$.

19. Jian L. Soy, isoflavones, and prostate cancer. Mol Nutr Food Res 2009; 53: 217-26.

20. Yang F, Song L, Wang H, et al. Quercetin in prostate cancer: Chemotherapeutic and chemopreventive effects, mechanisms and clinical application potential (Review). Oncol Rep 2015; 33: 2659-68.
21. Bosetti C, Bravi F, Talamini R, et al. Flavonoids and prostate cancer risk: a study in Italy. Nutr Cancer 2006; 56: $123-7$.

22. Sak K. Cytotoxicity of dietary flavonoids on different human cancer types. Pharmacogn Rev 2014; 8: 122-46.

23. Park SY, Murphy SP, Wilkens LR, et al. Legume and isoflavone intake and prostate cancer risk: The Multiethnic Cohort Study. Int J Cancer 2008; 123: 927-32.

24. Travis RC, Spencer EA, Allen NE, et al. Plasma phytooestrogens and prostate cancer in the European Prospective Investigation into Cancer and Nutrition. Br J Cancer 2009; 100: $1817-23$.

25. Ozasa K, Nakao M, Watanabe Y, et al. Serum phytoestrogens and prostate cancer risk in a nested case-control study among Japanese men. Cancer Sci 2004; 95: 65-71.

26. Guo K, Liang Z, Liu L, et al. Flavonoids intake and risk of prostate cancer: a meta-analysis of observational studies. Andrologia 2016; 48: 1175-82.

27. Strom SS, Yamamura Y, Duphorne CM, et al. Phytoestrogen intake and prostate cancer: a case-control study using a new database. Nutr Cancer 1999; 33: 20-5.

28. Hedelin M, Bälter KA, Chang ET, et al. Dietary intake of phytoestrogens, estrogen receptor-beta polymorphisms and the risk of prostate cancer. Prostate 2006; 66: 1512-20.

29. Ward HA, Kuhnle GG, Mulligan AA, et al. Breast, colorectal, and prostate cancer risk in the European Prospective Investigation into Cancer and Nutrition-Norfolk in relation to phytoestrogen intake derived from an improved database. Am J Clin Nutr 2010; 91: 440-8.

30. Knekt $P$, Järvinen $R$, Seppänen $R$, et al. Dietary flavonoids and the risk of lung cancer and other malignant neoplasms. Am J Epidemiol 1997; 146: 223-30.

31. Knekt P, Kumpulainen J, Järvinen R, et al. Flavonoid intake and risk of chronic diseases. Am J Clin Nutr 2002; 76: $560-8$.

32. Mursu J, Nurmi T, Tuomainen TP, et al. Intake of flavonoids and risk of cancer in Finnish men: The Kuopio Ischaemic Heart Disease Risk Factor Study. Int J Cancer 2008; 123: 660-3.

33. Geybels MS, Verhage BA, Arts IC, et al. Dietary flavonoid intake, black tea consumption, and risk of overall and advanced stage prostate cancer. Am J Epidemiol 2013; 177: $1388-98$.

34. Ward H, Chapelais G, Kuhnle GG, et al. Lack of prospective associations between plasma and urinary phytoestrogens and risk of prostate or colorectal cancer in the European Prospective into Cancer-Norfolk study. Cancer Epidemiol Biomarkers Prev 2008; 17: 2891-4.

35. Travis RC, Allen NE, Appleby PN, et al. Prediagnostic concentrations of plasma genistein and prostate cancer risk in 1605 men with prostate cancer and 1697 matched control participants in EPIC. Cancer Causes Control 2012; 23: $1163-71$.

36. Jackson MD, McFarlane-Anderson ND, Simon GA, et al. Urinary phytoestrogens and risk of prostate cancer in Jamaican men. Cancer Causes Control 2010; 21: 2249-57.

37. Park SY, Wilkens LR, Franke AA, et al. Urinary phytoestrogen excretion and prostate cancer risk: a nested case-control study in the Multiethnic Cohort. Br J Cancer 2009; 101: 185-91.

38. van Die MD, Bone KM, Williams SG, et al. Soy and soy isoflavones in prostate cancer: a systematic review and meta-analysis of randomized controlled trials. BJU Int 2014; 113: E119-30. 